\title{
Modeling and vibration decoupling control of multi-axial shaking table
}

\author{
Zhang Lianpeng ${ }^{1, a}$, Yang Zhidong ${ }^{1, b}$, Qu Zhiyong ${ }^{1, c}$, Han Junwei $^{1,2, d}$ \\ ${ }^{1}$ National State Key Laboratory of Robotics and System, Harbin Institute of Technology, Harbin, \\ China \\ ${ }^{2}$ Tianjin FYTY Technology Co., Tianjin, China \\ azlp_hit@163.com, byzd@hit.edu.cn, 'cqzy@hit.edu.cn, ${ }^{d}$ hjw@hit.edu.cn
}

\begin{abstract}
Keywords: electro-hydraulic shake table; waveform replication; decoupling control; frequency response function matrix; singular value decomposition
\end{abstract}

\begin{abstract}
Traditional control strategy study of the multi-axial electro-hydraulic shaking table(MAEHST) is based on linear model, ignoring the coupling and nonlinearity factors, which results in the waveform distortion and the control precision. To overcome these limitations, a model considering the hydraulic system nonlinearity and mechanical coupling using the software Simulink/SimMechanics is built. A vibration decoupling control strategy based on multiple input multiple output (MIMO) system identification is proposed to improve the waveform replication precision. The proposed control method applies the MIMO $H_{1}$ estimator to identify frequency response function matrix (FRFM) of the system, not only considering the diagonal element, but the off-diagonal element to acquire the coupling characteristics of six degrees of freedom (DOFs) of MAEHST. And then the inverse frequency response function matrix (IFRFM) is calculated using the singular value decomposition technique to obtain the drive signal combined with the reference signal. Simulation experiments are carried out, and the results indicate that the MIMO vibration decoupling control strategy successfully minimizes the coupling and improve the waveform replication precision of the shaking table.
\end{abstract}

\section{Introduction}

MAEHST remains irreplaceable position in the vibration test, which is the most reliable and efficient vibration and shock environment replication method to investigate the anti-seismic performance of various structures and articles ${ }^{[1-3]}$. In general, MAEHST system has eight or more cylinders to drive the platform to implement six DOFs motion. Thus, the cylinders need to coordinate to ensure the normal and accurate motion of the table. Even so, the cylinders still exist coupling each other during the motion, which results in the poor waveform control precision.

Many investigations ${ }^{[4-6]}$ have been carried out on the MAEHST vibration control strategy. Guan guangfeng ${ }^{[7]}$ developed the eight degree of freedom control technique and force balance controller to improve the control precision of the system. Yang zhidong ${ }^{[8]}$ researched on MIMO system identification method and proposed a $H_{v}$ technique to improve the system identification precision. $\mathrm{Yu}$ haoyang $^{[9]}$ proposed the EV system identification control strategy to implement the acceleration power spectrum replication. Shen gang ${ }^{[10]}$ put forward a method based on adaptive inverse control and an offline feed-forward compensator to obtain better replication accuracy. However, the algorithm ignores the coupling factor which will result in the poor control precision and coupling motion of unexpected DOFs.

Aimed at the problem of the poor vibration control precision of MAEHST system, this paper proposes a control strategy to compensate the system error which is caused by mechanical coupling. And the construction of this paper is organized as follows: Section two describes the MAEHST system including the servo control strategy, which is the foundation of the MAEHST control system. Section three studies vibration decoupling control algorithm by applying the MIMO system identification and IFRFM technique. Nonlinear hydraulic simulation model and mechanical model is 
built applying software Simulink/SimMechanics and simulation verification is processed in the section four. Section five concludes the main points and conclusion.

\section{The MAEHST system description and servo control system introduction}

As is shown, Fig.1 is the MAEHST diagram, which contains twelve identical hydraulic cylinders and servo valves. There is one acceleration sensor mounted at the platform as close as each piston rod of cylinder, and one LVDT displacement sensor mounted at each piston rod of cylinder. Twelve cylinders is driven to implement six DOFs of the platform. So the system is a hyper-redundant motion system with six redundant DOFs.

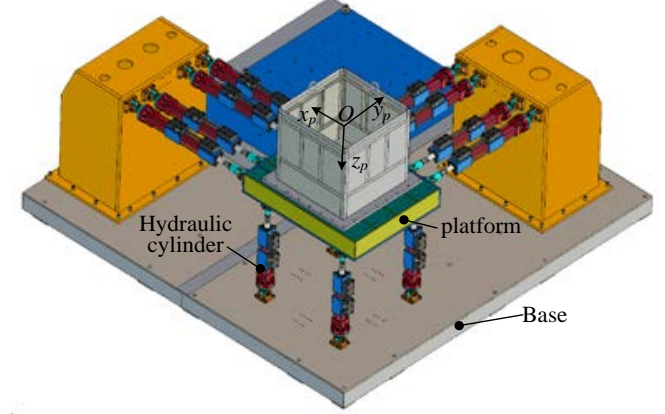

Fig.1 The schematic diagram of MAEHST Fig.2 The block diagram of MAEHST control system

Fig. 2 is the servo control system block diagram of MAEHST. The control system is comprised of signal generator unit, three variable controller, DOF synthesis unit, DOF decomposition unit, pressure balance controller and the platform and load unit. Three variable controller, which means displacement, velocity and acceleration variable, contains three variable feed-forward controller, aiming at broadening system frequency bandwidth, and three variable feedback controller, which is order to improve the stability of the control system. The pressure balance controller is used to reduce the inner force between the cylinders.

The hydraulic model of MAEHST mainly contains four parts: servo valve dynamics equation, servo valve flow equation, hydraulic cylinder flow continuity equation and force balance equation between the hydraulic cylinder and load. A Plummer has described model detailedly ${ }^{[11]}$, so this paper will not account for this part.

The six DOFs of the MAEHST is defined three translation motions along $X, Y, Z$ coordinate axial and three rotation motions around $X, Y, Z$ coordinate axial. According to the define and the geometry relation, the DOFs are followed by

$$
\begin{aligned}
& \mathrm{x}=\left(\mathrm{x}_{1}+\mathrm{x}_{2}+\mathrm{x}_{3}+\mathrm{x}_{4}\right) / 4 ; \\
& \mathrm{y}=\left(\mathrm{y}_{1}+\mathrm{y}_{2}+\mathrm{y}_{3}+\mathrm{y}_{4}\right) / 4 ; \\
& \mathrm{z}=\left(\mathrm{z}_{1}+\mathrm{z}_{2}+\mathrm{z}_{3}+\mathrm{z}_{4}\right) / 4 ; \\
& \mathrm{R}_{\mathrm{x}}=\left(-\mathrm{z}_{1}-\mathrm{z}_{2}+\mathrm{z}_{3}+\mathrm{z}_{4}\right) / 4 ; \\
& \mathrm{R}_{\mathrm{y}}=\left(-\mathrm{z}_{1}+\mathrm{z}_{2}-\mathrm{z}_{3}+\mathrm{z}_{4}\right) / 4 ; \\
& \mathrm{R}_{\mathrm{z}}=\left(\mathrm{x}_{1}+\mathrm{Kx}_{2}-\mathrm{Kx}_{3}-\mathrm{x}_{4}+\mathrm{y}_{1}+\mathrm{Ky}_{2}-\mathrm{Ky}_{3}-\mathrm{y}_{4}\right) / 8 ;
\end{aligned}
$$

Where, $K=L_{2} / L_{1}, L_{2}$ is the distance between $x_{1}$ and $x_{4}, L_{1}$ is the distance between $x_{2}$ and $x_{3}$.

So the DOF synthesis matrix is

$$
H_{h}=\left[\begin{array}{cccccccccccc}
3 & 3 & 3 & 3 & 0 & 0 & 0 & 0 & 0 & 0 & 0 & 0 \\
0 & 0 & 0 & 0 & 3 & 3 & 3 & 3 & 0 & 0 & 0 & 0 \\
0 & 0 & 0 & 0 & 0 & 0 & 0 & 0 & -3 & -3 & -3 & -3 \\
0 & 0 & 0 & 0 & 0 & 0 & 0 & 0 & 3 & -3 & -3 & 3 \\
0 & 0 & 0 & 0 & 0 & 0 & 0 & 0 & 3 & 3 & -3 & -3 \\
-2 & -1 & 1 & 2 & -2 & -1 & 1 & 2 & 0 & 0 & 0 & 0
\end{array}\right] / 12
$$

The DOF decomposition matrix is the pseudo inverse of the equation (2) 


$$
H_{f}=\left[\begin{array}{cccccccccccc}
1 & 1 & 1 & 1 & 0 & 0 & 0 & 0 & 0 & 0 & 0 & 0 \\
0 & 0 & 0 & 0 & 1 & 1 & 1 & 1 & 0 & 0 & 0 & 0 \\
0 & 0 & 0 & 0 & 0 & 0 & 0 & 0 & -1 & -1 & -1 & -1 \\
0 & 0 & 0 & 0 & 0 & 0 & 0 & 0 & 1 & -1 & -1 & 1 \\
0 & 0 & 0 & 0 & 0 & 0 & 0 & 0 & 1 & 1 & -1 & -1 \\
-1.2 & -0.6 & 0.6 & 1.2 & -1.2 & -0.6 & 0.6 & 1.2 & 0 & 0 & 0 & 0
\end{array}\right]^{T}
$$

Where, $[\cdot]^{\mathrm{T}}$ is the transpose of the matrix.

\section{Vibration decoupling control Algorithm}

In general, due to the nonlinearity of the control and the mechanical coupling between the cylinders, the waveform replication precision of MAEHST system is unsatisfied only using the servo control strategy. The former will cause the distortion of the waveform with harmonic waveforms disturbance, and the later will bring about the coupling motion of the unexpected DOFs.

To solve this problems, a vibration decoupling control technique is proposed combined with servo control strategy to improve the system control precision. And there are four important phases in the control algorithm: the signal generator for MIMO system identification, the MIMO FRFM identification technique, the calculation of MIMO IFRFM and the drive signal generation for the expected reference signal. The total process is

(1) Generator of the signal for MIMO system identification. For MIMO system with coupling each other, the drive signal must be uncorrelated to identify the coupling effect. So we apply the software MATLAB to generate six channel standard uniform random white noise signals $x(t)$, and the six channel is uncorrelated each other.

$$
x(t)=\operatorname{rand}(n, 6)
$$

(2) MIMO system identification. Driving the signals to the system, the sensors gather the six channel response signal $y(t)$, and The FRFM $H_{6 \times 6}(f)$ is calculated based on $H_{1}$ algorithm considering the off-diagonal effect.

$$
H_{6 \times 6}(f)=\left[H_{i j}(f)\right]_{6 \times 6}
$$

Where, $i=1 \sim 6, j=1 \sim 6$. DOFs.

The off-diagonal elements of $H_{6 X 6}(f)$ are not fully zeros due to the coupling effect between

(3) IFRFM. The IFRFM is the inverse of the FRFM, but the there will not exist inverse if the FRFM is singular, which is common in MAEHST system due to the coupling of nonlinear hydraulic system. If the FRFM is ill-conditioned, although the matrix is not singular, the system might be unstable. So the IFRFM must be processed in this condition. If the FRFM is not ill-conditioned, the IFRFM is

$$
H_{6 \times 6}^{-1}(f)=\operatorname{inv}\left(H_{6 \times 6}(f)\right)
$$

Where, $\operatorname{inv}(\cdot)$ is the inverse of a matrix.

If the FRFM is ill-conditioned, the IFRFM is

$$
H_{6 \times 6}^{-1}(f)=\operatorname{pinv}\left(H_{6 \times 6}(f)\right)
$$

Where, $\operatorname{pinv}(\cdot)$ is the Moore-Penrose pseudo-inverse with least squares sense.

(4) The drive signal for expected output signal. To get better response signal, which is well matched with the expected reference signal, the drive signal must be corrected according to the system frequency characteristic to reduce the effect of the MAEHST system.

Assumed that the reference signal is $r(t)$, the frequency spectrum of the $r(t)$ is obtained using the fast Fourier transform algorithm

$$
R(f)=F F T(r(t))
$$

Then the frequency spectrum $D(f)$ of the corrected drive signal $d(t)$ is

$$
D(f)=H_{6 \times 6}^{-1}(f) \cdot R(f)
$$

Applying the inverse fast Fourier transform calculate the corrected time drive signal $d(t)$ is 


$$
d(t)=\operatorname{IFFT}(D(f))
$$

Now, driving the corrected time drive signal $d(t)$ to the MAEHST, the system response signal will have better precision and smaller coupling motion, even to zeros.

\section{Modeling and simulation Experimental Verification of decoupling control algorithm}

The MAEHST system main parameters are listed in the Tab.1. The model of the system is divided into two main parts: hydraulic model and mechanical model. The hydraulic model is built using the software Simulink and the mechanical is built using the software SimMechanics to simulate the kinematics and dynamics of the MAEHST. The integral model built is shown as Fig.3, whose mechanical model part is displayed in Fig.4. The proposed algorithm will be verified through the simulation model.

Tab.1 Main parameters of Hyper-redundant MAEHST

\begin{tabular}{|c|c|}
\hline \\
\hline Parameters & Value(Unit) \\
\hline Mass of table and load & 1845(Kg) \\
\hline Nature frequency of servovalve & $120(\mathrm{~Hz})$ \\
\hline Damping ratio of servovalve & 0.6 \\
\hline Viscous damping coefficient & $10000(\mathrm{~N} /(\mathrm{m} / \mathrm{s}))$ \\
\hline Effective Piston area & $19\left(\mathrm{~m}^{2}\right)$ \\
\hline Hdraulic oil density & $845\left(\mathrm{Kg} / \mathrm{m}^{3}\right)$ \\
\hline Hydraulic power pressure & $1.8 \times 10^{7}(\mathrm{~Pa})$ \\
\hline Effective bulk modulus & $6.9 \times 10^{8}(\mathrm{~Pa})$ \\
\hline
\end{tabular}

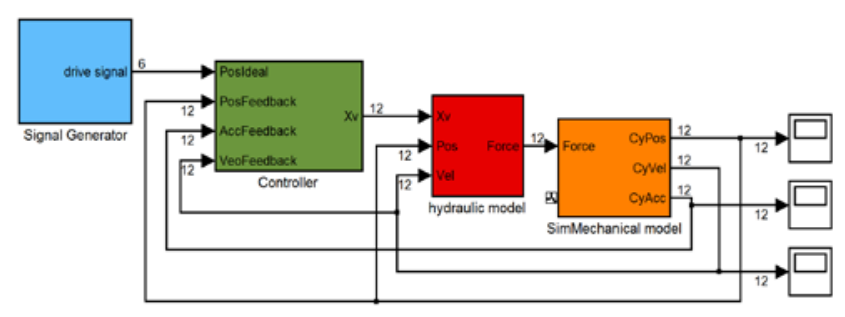

Fig.3 Control simulation model of MAEHST

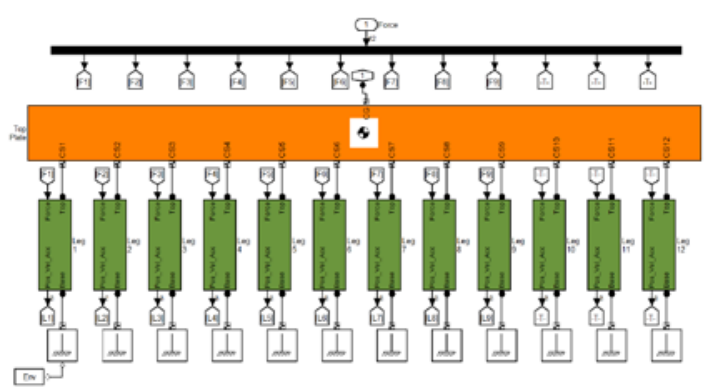

Fig.4 SimMechanics model of mechanical system

The expected reference signal of $X$ translation DOF is a 40s time random signal with $80 \mathrm{~Hz}$ frequency width. The reference signal and response signal with only servo control strategy are shown in Fig.5. And the coupling motions of other five DOFs are displayed in Fig.6. To convenient analysis, the coupling motion values of rotation DOFs are expressed using cylinders displacement of each DOF.

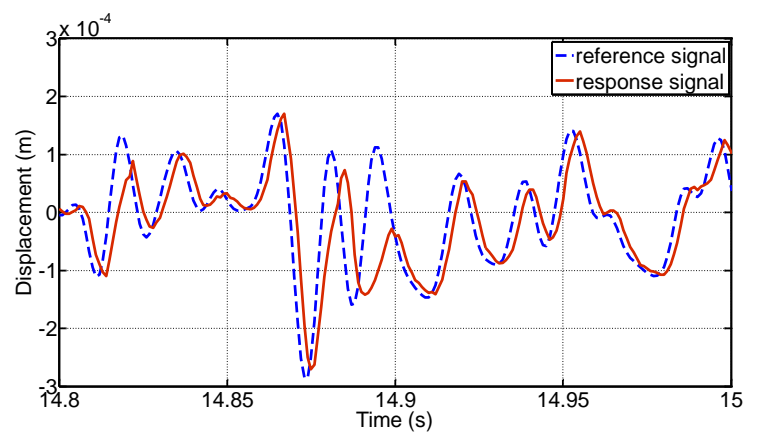

Fig.5 Reference curve and response curve of $\mathrm{X}$ DOF
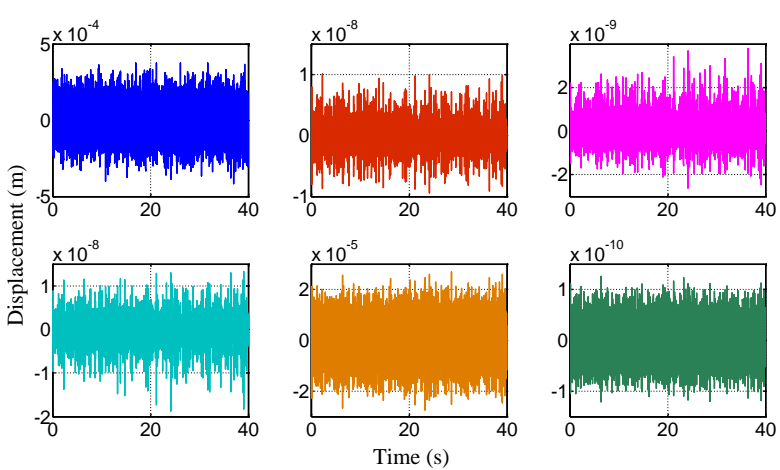

Fig.6 Coupling motion displacements under X DOF reference signal 
From the Fig.5 and Fig.6, we can see that the response signal cannot follow the reference signal very well and the deviation is quite large. The biggest coupling motion displacement is up to $0.02 \mathrm{~mm}$ to the reference signal $0.4 \mathrm{~mm}$, which is unacceptable for application. And the Ry DOF coupling motion displacement is relatively larger, because coupling between both is larger due to mechanical factor.

Fig.7 is the amplitude-frequency curves of MAEHST FRFM identified using the $H_{1}$ identification algorithm. Fig.8 is the amplitude-frequency curves of MAEHST IFRFM. As is shown, the off-diagonal elements are not zeros, which indicate that there are coupling between DOFs.

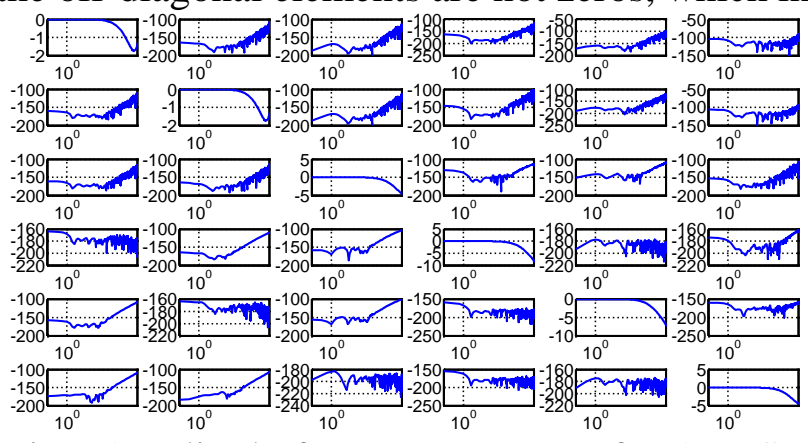

Fig.7 Amplitude-frequency curves of MAEHST system FRFM
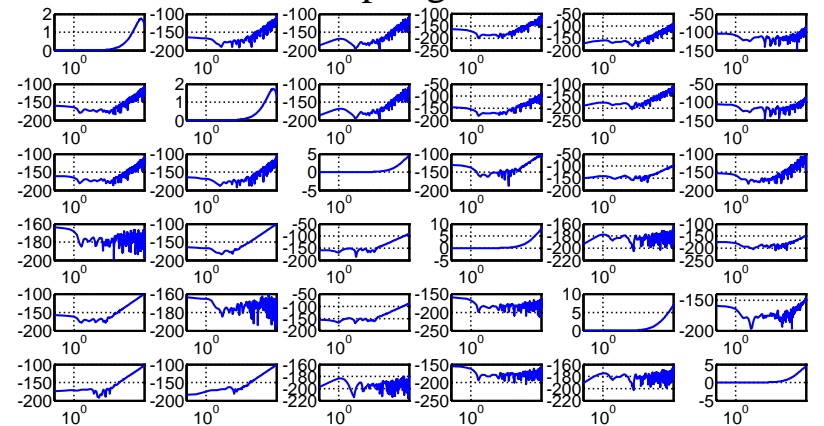

Fig.8 Amplitude-frequency curves of MAEHST system IFRFM

Fig.9 is the reference signal and the response signal of $X$ translation DOF with the decoupling control strategy proposed above. Fig.10 is the coupling motions of other DOFs with decoupling control algorithm.

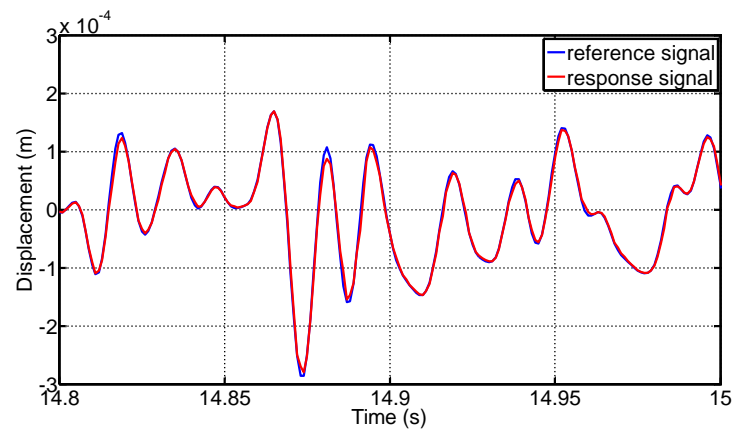

Fig.9 Reference curve and response curve of $\mathrm{X}$ DOF
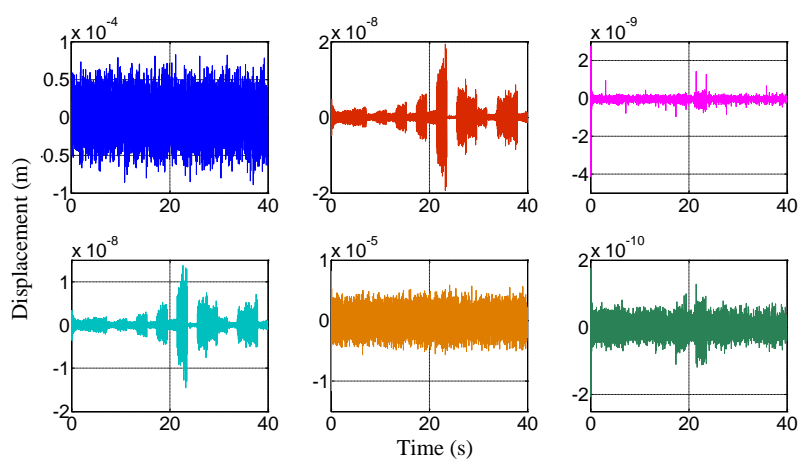

Fig.10 Coupling motion displacements under X DOF reference signal

From Fig.9 and Fig.10, it can be seen that the response signal can follow the reference signal very well with little error. The Ry DOF coupling displacement reduces tremendously, and the others also reduce comparing to the former. The biggest coupling motion displacement is $0.005 \mathrm{~mm}$ to the reference signal $4 \mathrm{~mm}$, which means the control algorithm proposed above reduces the coupling motion displacements and the decoupling control strategy is effective.

\section{Conclusion}

This paper proposed a vibration decoupling control strategy aimed at the disadvantage of waveform replication experiment of the MAEHST. The vibration decoupling technique was introduced and the deduced equations were given. The nonlinear simulation model of the six DOFs MAEHST of the IEST in Harbin Institute of technology was built using the software Simulink and SimMechanics. Simulation experiments were implemented using the built model to verify the proposed control strategy. The simulation results proved that control precision were unsatisfied and there bigger coupling motions and the error reduced to the meeting requirement with the vibration decoupling compensation control. Thus the proposed decoupling control algorithm is applicable and valid. 


\section{Acknowledgment}

This research was supported by the National Natural Science Foundation of China(Grant number 51205077) and was supported by the National Natural Science Foundation of China(Grant number 51475116) and also was supported by the Fundamental Research Funds for the Central Universities (Grant No. HIT.NSRIF.2015051) . The author would like to thank IEST and Dr. Zhidong Yang, Dr. Qu Zhiyong and Han Junwei of the Mechanical and Electrical Engineering, Harbin Institute of Technology for their assistance and appreciate the referees for their careful review.

\section{References}

[1] R.T.Severn: The development of shaking tables-a historical note. Earthquake engineering \& structural dynamics, Vol.41(2011), p.195-213.

[2] Andrew Plummer: A general co-ordinate transformation framework for multi-axis motion control with application in the test industry. Control engineering practice, Vol.18(2010) , p.598-607.

[3] Underwood M A, Keller T: Rectangular control of multi-shaker systems: theory and practical results. Journal of the Institute of Environment Sciences and Technology, Vol.1(2004) , p.80-86.

[4] Chen Jiayan, Wang Haidong, Chen Zhangwei: Progress in multi-exciter vibration testing control technology. Journal of vibration and shock, Vol.30(2001) , p.69-73.

[5] Hamma G A, Stroud R C, Underwood M A, et al: A review of multiaxis/multiexciter vibration technology. Sound and Vibration Mag, Vol.30(1996), p.20-27.

[6] A R Plummer: Control techniques for structural testing: a review. Proc Instn Mech Entgrs Part I; J Systems and control engineering, Vol.221(2007) , p.139-169.

[7] Guan Guangfeng, Xiong Wei, Han Junwei: Control strategy of a 6-DOF hydraulic shaker. Journal of vibration and shock, Vol.29(2010) , p.200-203.

[8] Yang Zhidong, Guan Guangfeng, Han Junwei, Li Hongren: Frequency response function for three axes hydraulic shaking system by using Hv algorithm. Journal of earthquake engineering and engineer vibration, Vol.27(2007), p.83-87.

[9] Yu Haoyang, Guan Guangfeng: Random vibration control algorithm of electro-hydraulic shaking table. Journal of heilongjiang institute of technology, Vol.26(2012), p.42-44.

[10] Shen Gang, Yang Chifu, Zhao Jinsong, Han Junwei: Adaptive feed-forward compensation for hybrid control with acceleration time waveform replication on electro-hydraulic shaking table. Control engineering practice, Vol.21(2013) , p.1128-1142.

[11]A.R.Plummer: A Detailed dynamic model of a six-axis shaking table. Journal of earthquake engineering, Vol.12(2008), p.631-662. 